ISSN 2525-4804

\title{
PRODUÇÃO DE SORGO SACARINO BRS 506 EM FUNÇÃO DO MANEJO DA ADUBAÇÃO: ÉPOCA DE CORTE PARA COLHEITA E IRRIGAÇÃO
}

\author{
Ruana Iris Fernandes Cruz ${ }^{1}$, Luis Fernando Vieira da Silva ${ }^{2}$, Antonio Alves Pinto ${ }^{3}$, Leandro Alves \\ Pinto $^{4}$, Felipe Thomaz da Camara ${ }^{5}$
}

\section{RESUMO:}

O sorgo sacarino (Sorghum bicolor L. Moench) vem se destacando na agroindústria como fonte promissora para produção de etanol devido ao seu alto teor de açúcar. Com isso, surge a necessidade de realizar mais estudos para avaliar as melhores formas de manejo da cultura. Objetivou-se avaliar as formas de manejo quanto ao parcelamento de adubação, época de corte para colheita e irrigação do sorgo sacarino, a fim de se buscar um arranjo que promova maior produtividade. O experimento foi conduzido a campo com delineamento em blocos casualizados, em esquema de parcelas sub-subdivididas, com três repetições. Utilizou-se duas épocas de corte, de 90 e 120 dias após a semeadura (DAS), dois manejos de irrigação, 90 e 45 minutos diários, iniciados a partir da floração e adubação nitrogenada de cobertura com uma, duas e três adubações. Foram avaliados altura das plantas, diâmetro do colmo, massa verde de colmo e massa verde total, teor de sólidos solúveis, volume de caldo, volume sólidos solúveis, massa seca de colmo e massa seca total. A permanência das plantas em campo por mais 30 dias resulta em aumento da altura, massa verde de colmo e teor de sólidos solúveis. O tempo de irrigação de 45 minutos diários é suficiente para suprir a necessidade da cultura do sorgo em cultivos com condições similares a deste trabalho. O parcelamento da adubação de cobertura em três vezes não resulta em aumento significativo que justifique a adesão dessa prática, sendo recomendado o parcelamento em duas vezes com resultados similares ao parcelamento em três vezes, porém com menor custo de mão de obra no final do ciclo.

Palavras-chave: biomassa, estresse hídrico, etanol.

\section{PRODUCTION OF SACARINE SORGHUM BRS 506 IN FUNCTION OF THE MANAGEMENT OF FERTILIZATION, CUTTING TIME FOR HARVESTING AND IRRIGATION}

\footnotetext{
${ }^{1}$ Universidade Federal Rural de Pernambuco (UFRPE), Recife, PE. E-mail: ruanaiiriss@gmail.com

${ }^{2}$ Laboratório de Mineralogia do Solo, Escola Superior de Agricultura Luiz de Queiroz (ESALQ/USP), Universidade de São Paulo, Piracicaba, SP. E-mail: vieira.silva@usp.br

${ }^{3}$ Laboratório de Instrumentação e Agricultura de Precisão, Universidade estadual Paulista (UNESP/FCAV), Jaboticabal, SP. E-mail: alves.pinto@unesp.br

${ }^{4}$ Universidade Estadual Paulista (UNESP/FCAV), Jaboticabal, SP. E-mail: leandro.alves-pinto@ @unesp.br

${ }^{5}$ Universidade Federal do Cariri (UFCA), Crato, CE. E-mail: antonioufca@gmail.com
} 
ISSN 2525-4804

\section{ABSTRACT:}

Sweet sorghum (Sorghum bicolor L. Moench) is standing out in the agroindustry, as a promising source for ethanol production due to its high sugar content, with this there is a need to conduct further studies to evaluate the best ways of crop management. The objective of this study was to evaluate the ways of management regarding the spreading of fertilization, cutting season for harvesting and irrigation of sweet sorghum, in order to seek an arrangement that promotes greater productivity. The experiment was conducted in the field with a randomized block design, in a subdivided plot scheme, with three replications. Two cutting seasons were used, 90 days and 120 days after sowing (DAS), two irrigation managements, 90 and 45 minutes daily, starting from flowering and covering nitrogen fertilization, two and three fertilizations. Plant height, stalk diameter, green stalk mass, total green mass, soluble solids content, broth volume, and soluble solids volume, stalk dry mass and total dry mass. The permanence of the plants in the field for 30 days more results in increase height, green stalk mass and soluble solids content. The irrigation time of 45 minutes daily is enough to supply the need for the cultivation of sorghum in crops with conditions similar to this work. The division of covering fertilization in three times does not result in a significant increase that justifies the adherence of this practice, being recommended the division in two times because the results are similar to the division in three times, but with lower labor cost at the end of the cycle.

Keywords: biomass, water stress, ethanol. 


\section{INTRODUÇÃO}

Com o aumento da demanda mundial por combustíveis, intensifica-se a busca por fontes alternativas, como plantas aquáticas, culturas celulósicas (resíduos vegetais e madeira) e culturas agrícolas (Godwin et al., 2019; Stamenkovic et al., 2020). Os biocombustíveis (bioetanol e biodiesel), produzidos a partir de fontes renováveis, estão ganhando cada vez mais importância em função do aumento dos preços dos combustíveis fósseis convencionais, esgotamento das reservas petrolíferas e a preocupação em relação aos gases de efeito estufa. Segundo Masiero et al. (2008), a produção de combustíveis através da biomassa de gramíneas como o sorgo, apresenta-se como uma alternativa viável e atrativa para indústria automobilística.

No Brasil, de acordo com Prado et al. (2019), o sorgo é amplamente utilizado na alimentação animal, todavia pesquisas apontam sobre viabilidade e o potencial fisiológico das espécies para a utilização na produção de combustível. Segundo Silva et al. (2020) o sorgo sacarino (Sorghum bicolor L. Moench) destaca-se por ser uma espécie de comportamento rústico, alta adaptabilidade aos diferentes climas do país e do mundo, podendo ser cultivado em clima tropical, subtropical e temperado. Apresenta elevada tolerância a períodos de estiagem durante o ciclo vital, podendo ser cultivado desde solos argilosos até ligeiramente arenosos, porém desenvolve-se melhor em solos bem preparados (Leite et al., 2012). Entretanto, deve-se atentar ao manejo correto da cultura buscando sempre maximizar a sua eficiência.

Um conhecimento completo do manejo mais eficiente desta planta deve ter como um dos fatores em destaque, principalmente para regiões de clima quente e seco como o semiárido brasileiro, o manejo hídrico (Carmo et al., 2020; Hassan et al., 2020). Apesar de ter ciência da resistência do sorgo sacarino à seca, ainda há carência de trabalhos que demostrem a magnitude do impacto que o estresse hídrico pode provocar no cultivo de sorgo, seja na produção de etanol, ou na produção de biomassa (Bell et al., 2020; Mohammed et al., 2020). Sabe-se que o sorgo apresenta menos necessidade de água e adubo do que outras culturas semelhantes, como o milho; no entanto, nota-se que há poucos estudos que analisam os efeitos da adubação nitrogenada na produção de biomassa e seus efeitos sobre o brix e rendimento de açúcares (Morey et al., 2018; Silva et al., 2019).

Segundo Disasa et al. (2018), pouco se sabe sobre o mecanismo de acumulação de açúcar nos colmos do sorgo sacarino; contudo, sabe-se que em culturas como o sorgo e cana-de-açúcar, a época de colheita tem especial destaque em um manejo eficiente. Dessa forma, objetivou-se avaliar as formas de manejo quanto ao parcelamento de adubação, época de corte para colheita e irrigação do sorgo sacarino, a fim de se buscar um arranjo que promova maior produtividade.

\section{MATERIAL E MÉTODOS}

O experimento foi conduzido na área experimental da Universidade Federal do Cariri (UFCA), Crato-CE, localizada na latitude de $7^{\circ} 14^{\prime}$ $3,4^{\prime \prime} \mathrm{S}$ e longitude de $39^{\circ} 22^{\prime} 7,6^{\prime \prime} \mathrm{W}$, com altitude média de $442 \mathrm{~m}$. O clima da região é o Aw, caracterizado como clima tropical úmido com estação seca no inverno, apresentando temperatura média anual de $27^{\circ} \mathrm{C}$ (Lima et al., 2017). O solo foi classificado como ARGISSOLO VERMELHOAMARELO, conforme descrito por Santos et al. (2018).

O solo foi coletado na profundidade $0,0-$ 0,20 m, para realização das análises químicas, cujas propriedades apresentaram os seguintes resultados: pH em água: 4,3 $\mathrm{P}$ (mehlich1): $7.0 \mathrm{mg} \mathrm{dm}^{-3}$; $\mathrm{MO}$ : $2,28 \mathrm{~g} \mathrm{~kg}^{-1}$; $\mathrm{e} \mathrm{H}^{+} \mathrm{Al}^{3+}, \mathrm{K}^{+}, \mathrm{Ca}^{2+}, \mathrm{Mg}^{2+}, \mathrm{Na}^{+}$e $\mathrm{Al}^{3+}$. 1,98 cmol $_{\mathrm{c}} \mathrm{kg}^{-1} ; 0,08 \mathrm{cmol}_{\mathrm{c}} \mathrm{kg}^{-1} ; 0,2$ cmol $_{\mathrm{c}} \mathrm{kg}^{-1} ; 0,3$ $\mathrm{cmol}_{\mathrm{c}} \mathrm{kg}^{-1} ; 0,06 \mathrm{cmol}_{\mathrm{c}} \mathrm{kg}^{-1}$; e 0,7 cmol $_{\mathrm{c}} \mathrm{kg}^{-1}$, respectivamente e $\mathrm{V} \%: 23$.

$\mathrm{O}$ delineamento experimental utilizado foi em blocos casualizados, em esquema de parcelas 
sub-subdivididas, com três repetições, totalizando 36 unidades experimentais. As parcelas foram constituídas por duas épocas de corte das plantas, sendo a primeira realizada aos 90 dias após a semeadura (DAS) e a segunda aos 120 (DAS). As subparcelas foram constituídas por dois manejos de irrigação, o primeiro com turno de rega de $1 \mathrm{~h}$ e 30 min por dia e o segundo com turno de rega de 45 min diários, iniciados a partir da floração. As subsubparcelas consistiram no número de adubações de cobertura nitrogenada $(1-$ uma cobertura nitrogenada, 2 - duas coberturas nitrogenadas e 3 três coberturas nitrogenadas) realizadas em intervalos de 15 dias entre elas, sendo a primeira parcela aplicada com 21 DAS e as demais com $42 \mathrm{e}$ 63 DAS.

Cada unidade experimental ocupou uma área de $16,5 \mathrm{~m}^{2}(5,5 \times 3,0 \mathrm{~m})$, sendo que a parcela foi composta por três fileiras de plantas, espaçadas a 1,0 $\mathrm{m}$ entre si, com 5,5 $\mathrm{m}$ de comprimento, e as plantas espaçadas a $0,10 \mathrm{~m}$, totalizando uma população de 100.000 plantas por hectare. A área que correspondia ao ensaio foi toda demarcada e piqueteada para facilitar a aplicação dos tratamentos, semeadura e instalação do sistema de irrigação. A parcela útil para a análise foi de $2 \mathrm{~m}^{2}$, sendo avaliada a fileira central eliminando-se a bordadura.

A adubação foi realizada com base na análise de solo e nas exigências da cultura, utilizando-se a recomendação feita pela Embrapa (2012). Utilizou-se como dose ideal (100\%) as quantias de $30 \mathrm{~kg} \mathrm{ha}^{-1}$ de $\mathrm{N}\left(150 \mathrm{~kg} \mathrm{ha}^{-1}\right.$ de sulfato de amônio), $80 \mathrm{~kg} \mathrm{ha}^{-1}$ de $\mathrm{P}_{2} \mathrm{O}_{5}$ (445 $\mathrm{kg} \mathrm{ha}^{-1} \mathrm{de}$ superfosfato simples) e $50 \mathrm{~kg} \mathrm{ha}^{-1} \mathrm{de}_{2} \mathrm{O}$ (83 kg ha1 de cloreto de potássio) na semeadura. Para cobertura foram utilizadas as quantias de $100 \mathrm{~kg}$ ha ${ }^{1}$ de N (500 kg ha ${ }^{-1}$ de sulfato de amônio).

$\mathrm{O}$ sistema de irrigação foi por mangueira gotejadora, com vazão de $5,0 \mathrm{~L} \mathrm{~h}^{-1} \mathrm{~m}^{-1}$, com irrigação diária por $1,5 \mathrm{~h}$ até $\mathrm{o}$ início do florescimento (pendoamento) do sorgo sacarino, momento no qual foram aplicados os tratamentos, referentes às subparcelas, com tempo de irrigação de $1,5 \mathrm{~h}$ e $0,75 \mathrm{~h}$, portanto, totalizando $560 \mathrm{~mm} \mathrm{e}$ $670 \mathrm{~mm}$ de irrigação para colheita realizado com 90 DAS para os dois tratamentos, respectivamente 1,5 h e 0,75 h. Já para irrigações com colheita realizada em 120 dias após a semeadura, foram utilizadas lâminas de $670 \mathrm{~mm}$ para regime de 45 minutos diários de irrigação e $900 \mathrm{~mm}$ de irrigação para regime de 90 minutos diários.

A variedade utilizada foi o sorgo sacarino BRS 506, tolerante a seca e ciclo de 130 dias após a emergência, o qual apresenta rendimento médio de $50 \mathrm{t} \mathrm{ha}^{-1}$ de colmo, com alto teor de açúcares, principalmente sacarose (Embrapa, 2012). A operação de semeadura foi realizada no dia 14 de setembro de 2017, depositando as sementes em sulcos espaçados a $0,8 \mathrm{~m}$ entre si, com profundidade de $0.1 \mathrm{~m}$.

Após a colheita foi avaliada a altura de plantas (AP) (determinada a partir da base do caule "coleto" até o ápice da folha mais velha), diâmetro do colmo (DC) (determinado com o auxílio de um paquímetro em todas as plantas colhidas na parcela útil, sendo realizado na base do caule). Os colmos foram prensados em moenda manual e o caldo recolhido em recipiente com a finalidade de se determinar o volume de caldo (VC) e o volume sólidos solúveis (VSS) para aferição do teor de sólidos solúveis (SS) foi utilizado refratômetro analógico. Em seguida, determinou-se a massa verde de colmo (MVC), massa verde total (MVT). Posteriormente, as plantas foram secas em estufa a $65^{\circ} \mathrm{C}$ até massa constante, visando à determinação da massa seca de colmo (MSC) e massa seca total (MST).

Os resultados foram submetidos à análise de variância pelo teste $\mathrm{F}$, e quando significativas as médias foram comparadas pelo teste de Tukey a 5\% de probabilidade, utilizando o software Sisvar (Ferreira, 2011). As correlações foram realizadas empregando-se os coeficientes de correlação de Pearson, utilizando o software R (R Core Team, 2016).

\section{RESULTADOS E DISCUSSÃO}


Verifica-se que os cortes apresentaram diferença significativa $(\mathrm{p}<0,01)$ para a altura das plantas (AP) e massa verde total (MVT), sendo que os maiores valores AP e MVT foram no segundo corte (120 DAS) 1,92 m e $43622 \mathrm{~kg} \mathrm{ha}{ }^{-1}$, respectivamente. Provavelmente, em função dos 30 dias a mais que a planta teve para crescer aumentando a biomassa das plantas. Para a altura das plantas e diâmetro dos colmos (DC) os coeficientes de variação foram de baixos a médios e de médios a altos para a massa verde do colmo (MVC) e MVT, conforme a classificação de Pimentel-Gomes (2009).

Tabela 1 - Síntese da análise de variância e do teste de médias para a altura das plantas (AP), o diâmetro do colmo (DC), a massa verde de colmos (MVC) e a massa verde total (MVT).

\begin{tabular}{|c|c|c|c|c|}
\hline \multirow{2}{*}{ Fontes de Variação } & \multicolumn{4}{|c|}{ Valores de F } \\
\hline & $\mathbf{A P}$ & DC & MVC & MVT \\
\hline Cortes $(\mathrm{C})$ & $26,42^{*}$ & $8,54^{\mathrm{NS}}$ & $10,18^{\mathrm{NS}}$ & $11,10^{*}$ \\
\hline Irrigação (I) & $0,03^{\mathrm{NS}}$ & $0,56^{\mathrm{NS}}$ & $0,20^{\mathrm{NS}}$ & $0,17^{\mathrm{NS}}$ \\
\hline Interação CXI & $0,34^{\mathrm{NS}}$ & $2,64^{\mathrm{NS}}$ & $0,47^{\mathrm{NS}}$ & $0,59^{\mathrm{NS}}$ \\
\hline Adubação (A) & $5,11^{*}$ & $0,87^{\mathrm{NS}}$ & $2,65^{*}$ & $2,78^{*}$ \\
\hline Interação $\mathrm{C} \times \mathrm{A}$ & $0,71^{\mathrm{NS}}$ & $4,09^{*}$ & $0,13^{\mathrm{NS}}$ & $0,14^{\mathrm{NS}}$ \\
\hline Interação I×A & $4,63^{*}$ & $2,16^{\mathrm{NS}}$ & $0,73^{\mathrm{NS}}$ & $0,70^{\mathrm{NS}}$ \\
\hline Interação $\mathrm{C} \times \mathrm{I} \times \mathrm{A}$ & $0,75^{\mathrm{NS}}$ & $3,36^{\mathrm{NS}}$ & $0,78^{\mathrm{NS}}$ & $0,68^{\mathrm{NS}}$ \\
\hline CV $1(\%)$ & 2,79 & 8,52 & 10,99 & 9,50 \\
\hline CV $2(\%)$ & 3,90 & 9,97 & 30,72 & 30,26 \\
\hline CV $3(\%)$ & 6,81 & 10,00 & 24,53 & 24,37 \\
\hline \multirow{2}{*}{ Fatores } & \multicolumn{4}{|c|}{ Teste de Médias de Tukey $(\mathrm{p}<0,05)$} \\
\hline & $\mathbf{A P}$ & DC & MVC & MV \\
\hline Corte $(\mathrm{C})$ & \multicolumn{2}{|c|}{ 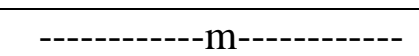 } & \multicolumn{2}{|c|}{--------kg ha-1-------- } \\
\hline Primeiro & $1,83 \mathrm{~b}$ & $0,017 \mathrm{a}$ & $32206 a$ & $39250 \mathrm{~b}$ \\
\hline Segundo & $1,92 \mathrm{a}$ & $0,019 \mathrm{a}$ & $36206 \mathrm{a}$ & $43622 \mathrm{a}$ \\
\hline \multicolumn{5}{|l|}{ Irrigação (I) } \\
\hline $45 \mathrm{~min}$ & $1,88 \mathrm{a}$ & $0,018 \mathrm{a}$ & $33409 a$ & $40571 \mathrm{a}$ \\
\hline $90 \mathrm{~min}$ & $1,88 \mathrm{a}$ & $0,018 \mathrm{a}$ & $35003 \mathrm{a}$ & $42301 \mathrm{a}$ \\
\hline \multicolumn{5}{|l|}{ Adubação (A) } \\
\hline 1 & $1,78 b$ & $0,018 \mathrm{a}$ & $29890 \mathrm{~b}$ & $35927 \mathrm{~b}$ \\
\hline 2 & $1,94 \mathrm{a}$ & $0,018 \mathrm{a}$ & 37636 a & $45124 \mathrm{a}$ \\
\hline 3 & $1,91 \mathrm{ab}$ & $0,017 \mathrm{a}$ & $35092 \mathrm{ab}$ & $43257 \mathrm{ab}$ \\
\hline
\end{tabular}

Médias seguidas pela mesma letra minúscula na coluna, não diferem entre si pelo teste de Tukey a 5\% de probabilidade. **: significativo $(\mathrm{P}<0,01)$; *: significativo $(\mathrm{P}<0,05)$; NS: não significativo; CV\%: coeficiente de variação.

Para o fator parcelamento da adubação ocorreu significância $(\mathrm{p}<0,05)$ para AP, MVC e MVT, no parcelamento em uma e duas vezes, sendo os maiores valores com o parcelamento em duas vezes, porém não diferindo estatisticamente do parcelamento em três vezes. $\mathrm{O}$ acréscimo de mais parcelamento resulta em mais custos de produção, o que coloca o parcelamento em duas vezes como a melhor opção para o produtor.
Com base nos resultados obtidos da análise de variância apresentados na Tabela 1, observa-se que ocorreu interação significativa entre o fator irrigação e adubação para altura das plantas, e para o diâmetro do colmo a interação foi entre os cortes e adubação, sendo essas interações desdobradas na Tabela 2.

Nota-se na Tabela 2 que na variável altura de planta o parcelamento da adubação 
nitrogenada em uma vez obteve o menor valor de altura $(1,70 \mathrm{~m})$, quando foi irrigado por apenas 45 minutos diários. Para a interação entre corte e adubação, ocorrida na variável diâmetro do colmo, o parcelamento em três vezes no primeiro corte obteve o menor valor significativo de diâmetro de colmo $(1,60 \mathrm{~cm})$.

Tabela 2 - Interação entre os fatores irrigação e adubação para a altura das plantas (AP) e entre os fatores corte e adubação para o diâmetro do colmo (DC).

\begin{tabular}{ccccc}
\hline \multirow{2}{*}{ Variável } & Fator & \multicolumn{3}{c}{ Adubação } \\
\cline { 2 - 5 } & Irrigação & & $\mathbf{2}$ & $\mathbf{3}$ \\
\hline \multirow{2}{*}{ Altura (m) } & $45 \mathrm{~min}$ & $1,70 \mathrm{bB}$ & $1,96 \mathrm{aA}$ & $1,99 \mathrm{aA}$ \\
& $90 \mathrm{~min}$ & $1,87 \mathrm{aA}$ & $1,93 \mathrm{aA}$ & $1,84 \mathrm{bA}$ \\
\hline \multirow{2}{*}{ Diâmetro (cm) } & Corte & & & \\
& Primeiro & $1,77 \mathrm{aA}$ & $1,91 \mathrm{aA}$ & $1,60 \mathrm{Bb}$ \\
& Segundo & $1,94 \mathrm{aA}$ & $1,84 \mathrm{aA}$ & $1,96 \mathrm{Aa}$ \\
\hline
\end{tabular}

Médias seguidas pela mesma letra minúscula nas colunas e maiúscula nas linhas, não diferem estatisticamente entre si segundo o teste de Tukey a 5\% de probabilidade.

Tabela 3 - Síntese da análise de variância e do teste de médias para o teor de sólidos solúveis (SS), o volume de caldo por hectare (VC), o volume de sólidos solúveis (VSS) massa seca de colmos (MSC), e a massa seca total (MST).

\begin{tabular}{|c|c|c|c|c|c|}
\hline \multirow{2}{*}{ Fontes de Variação } & \multicolumn{5}{|c|}{ Valores de F } \\
\hline & SS & VC & VSS & MSC & MST \\
\hline Corte (C) & $21,13^{*}$ & $12,32^{\mathrm{NS}}$ & $2,36^{\mathrm{NS}}$ & $2,97^{\mathrm{NS}}$ & $13,89^{\mathrm{NS}}$ \\
\hline Irrigação (I) & $12,46^{*}$ & $0,39^{\mathrm{NS}}$ & $0,16^{\mathrm{NS}}$ & $1,46^{\mathrm{NS}}$ & $0,02^{\mathrm{NS}}$ \\
\hline Interação CXI & $2,94^{\mathrm{NS}}$ & $0,07^{\mathrm{NS}}$ & $0,08^{\mathrm{NS}}$ & $0,41^{\mathrm{NS}}$ & $1,17^{\mathrm{NS}}$ \\
\hline Adubação (A) & $0,69^{\mathrm{NS}}$ & $3,57^{*}$ & $3,86^{*}$ & $10,33^{* *}$ & $2,72^{* *}$ \\
\hline Interação $\mathrm{C} \times \mathrm{A}$ & $1,93^{\mathrm{NS}}$ & $0,03^{\mathrm{NS}}$ & $0,00^{\mathrm{NS}}$ & $1,74^{\mathrm{NS}}$ & $0,34^{\mathrm{NS}}$ \\
\hline Interação I×A & $1,54^{\mathrm{NS}}$ & $0,05^{\mathrm{NS}}$ & $0,03^{\mathrm{NS}}$ & $0,56^{\mathrm{NS}}$ & $0,02^{\mathrm{NS}}$ \\
\hline Interação $\mathrm{C} \times \mathrm{I} \times \mathrm{A}$ & $0,88^{\mathrm{NS}}$ & $0,78^{\mathrm{NS}}$ & $1,03^{\mathrm{NS}}$ & $0,91^{\mathrm{NS}}$ & $0,62^{\mathrm{NS}}$ \\
\hline CV $1(\%)$ & 6,93 & 13,16 & 13,04 & 9,42 & 5,92 \\
\hline $\mathrm{CV} 2(\%)$ & 4,11 & 70,51 & 66,46 & 24,50 & 30,27 \\
\hline CV $3(\%)$ & 4,29 & 53,40 & 50,19 & 20,22 & 23,04 \\
\hline \multirow{2}{*}{ Fatores } & \multicolumn{5}{|c|}{ Teste de Médias de Tukey $(\mathrm{p}<0,05)$} \\
\hline & SS & $\mathrm{VC}$ & VSS & MSC & MST \\
\hline Corte (C) & ${ }^{\circ}$ Brix & \multicolumn{2}{|c|}{------ L ha-1------ } & \multicolumn{2}{|c|}{------kg ha'-1--- } \\
\hline Primeiro & $17,7 \mathrm{~b}$ & $6602 \mathrm{a}$ & $1167 \mathrm{a}$ & $4401 \mathrm{a}$ & $7142 \mathrm{a}$ \\
\hline Segundo & $19,7 \mathrm{a}$ & $5658 \mathrm{a}$ & $1092 \mathrm{a}$ & $4169 a$ & $7272 \mathrm{a}$ \\
\hline \multicolumn{6}{|l|}{ Irrigação (I) } \\
\hline $45 \min$ & $19,2 \mathrm{a}$ & $5679 a$ & $1079 a$ & $4073 a$ & $6956 a$ \\
\hline $90 \mathrm{~min}$ & $18,3 \mathrm{~b}$ & $6582 \mathrm{a}$ & $1180 \mathrm{a}$ & $4497 \mathrm{a}$ & $7459 a$ \\
\hline \multicolumn{6}{|l|}{ Adubação (A) } \\
\hline 1 & $18,9 \mathrm{a}$ & $4346 \mathrm{~b}$ & $815 \mathrm{~b}$ & $3693 \mathrm{~b}$ & $6289 \mathrm{~b}$ \\
\hline 2 & $18,8 \mathrm{a}$ & 7918 a & $1458 \mathrm{a}$ & $5201 \mathrm{a}$ & $8402 \mathrm{a}$ \\
\hline 3 & $18,5 \mathrm{a}$ & $6127 \mathrm{ab}$ & $1117 \mathrm{ab}$ & $3962 \mathrm{~b}$ & $6930 \mathrm{~b}$ \\
\hline
\end{tabular}

Médias seguidas pela mesma letra minúscula na coluna, não diferem entre si pelo teste de Tukey a 5\% de probabilidade. **: significativo $(\mathrm{P}<0,01)$; *: significativo $(\mathrm{P}<0,05)$; NS: não significativo; CV\%: coeficiente de variação. 
No fator corte e irrigação, observa-se que o teor de sólidos solúveis (SS) foi a única variável com significância a $5 \%$ de probabilidade, sendo que a permanência da planta em campo por mais 30 dias proporcionou aumento de $11,2 \%$ no teor de sólidos solúveis do sorgo sacarino, conforme descrito na Tabela 3.

Enquanto a redução de 30 minutos no tempo de irrigação resultou no aumento de $5 \%$ do SS, ou seja, quanto maior o índice de brix menos água e mais sólidos serão encontrados, corroborando com os estudos Isejima et al.

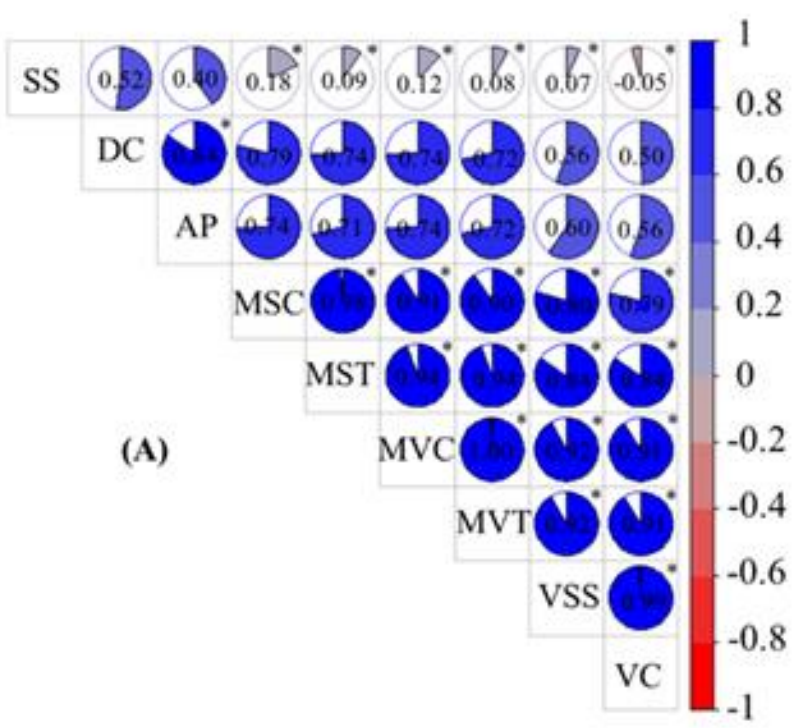

(2002) ao determinar açúcares redutores em cana-de-açúcar.

No fator adubação o volume de caldo (VC), o volume de sólidos solúveis (VSS), obtiveram significância a 5\%, e a massa seca de colmos (MSC) e massa seca total (MST) foram a $1 \%$ de probabilidade, sendo que o parcelamento da adubação nitrogenada em duas aplicações resultou nos melhores valores para ambas as variáveis.

A Figura 1 apresenta os coeficientes de correlação de Pearson entre todos os parâmetros investigados.

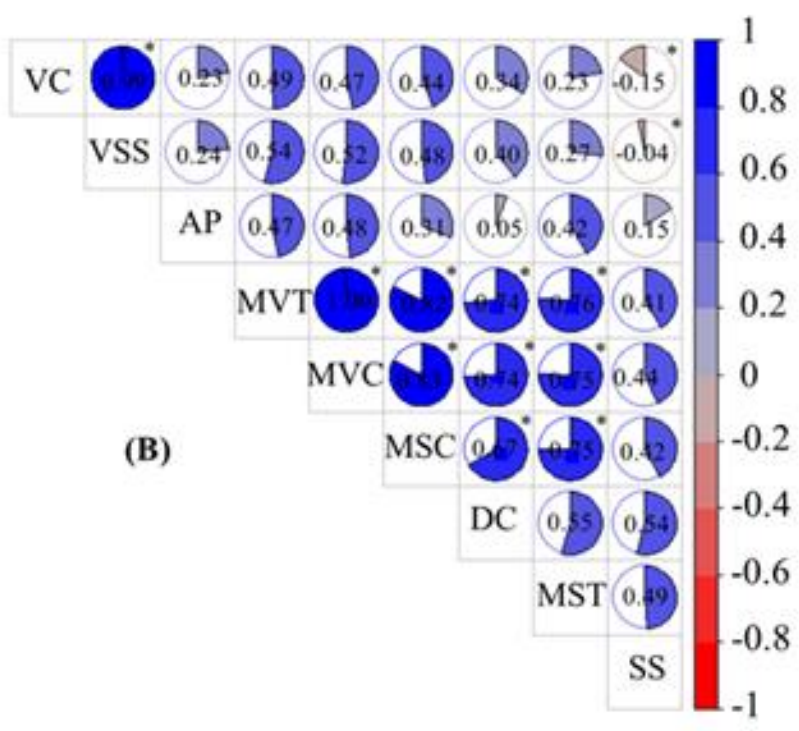

Figura 1 - Correlação entre parâmetros de desenvolvimento vegetativo de plantas. Primeiro corte (A) e segundo corte (B). ${ }^{*}$ significativo $(p<0,05)$.

De acordo com Devore (2006), ocorreu correlação negativa bem fraca e significativa entre o volume de caldo (VC) e teor de sólidos solúveis (SS) no primeiro e segundo corte ( $r=$ $0,05 ; r=-0,15 ; p<0.05)$ respectivamente. Isso pode ser consequência da conservação póscolheita dos colmos do sorgo sacarino, corroborando com os resultados obtidos por Sobreira et al. (2009) ao estudarem características de conservação pós-colheita de frutos de tomateiro. De acordo com Leite et al.
(2019), os teores de sólidos solúveis são importantes para conseguir um caldo com elevada qualidade de fermentação e, consequentemente, maximizar a produção de etanol por hectares.

Ao analisar as correlações entre teor de sólidos solúveis (SS) e altura de plantas (AP) no primeiro e segundo corte, observamos correlações positivas fraca $(r=0,40 ; r=0,15)$ respectivamente; porém ocorreu uma redução entre os cortes. De acordo com Chi et al. (2020), 
esta diminuição é explicada devido a planta ficar mais tempo ao campo, reduzindo os teores de açúcar no colmo e, consequentemente, minimizando a produção de etanol por hectares. O peso da massa verde colmo (MVC) apresentou alta correlação positiva muito forte $\mathrm{e}$ significativa $(r=0,92 ; p<0.05)$ com o volume de sólidos solúveis (VSS) no primeiro corte, indicando que este caráter pode proporcionar ganho satisfatório no volume do caldo. Segundo Nur et al. (2019), está relação direta sobre (MVC), será eficiente para melhorar o volume do caldo.

\section{CONCLUSÃO}

A permanência das plantas em campo por mais 30 dias resulta em aumento da altura, massa verde de colmo e teor de sólidos solúveis.

O tempo de irrigação de 45 minutos diários é suficiente para suprir a necessidade da cultura do sorgo em cultivos com condições similares à deste trabalho.

O parcelamento da adubação de cobertura em três vezes não resulta em aumento significativo que justifique a adesão dessa prática, sendo recomendado o parcelamento em duas vezes com resultados similares ao parcelamento em três vezes, porém com menor custo de mão de obra no final do ciclo.

\section{REFERÊNCIAS BIBLIOGRÁFICAS}

Bell, J.M.; Schwartz, R.C.; Mclnnes, K.J.; Howell, K.J.; Morgan, C.L.S. (2020). Effects of irrigation level and timing on profile soil water use by grain sorghum. Agricultural Water Management. 232(1): 1-10.

Carmo, E.L.D.; Sousa, J.V.A.; Ferreira, C.J.B.; Braz, G.B.P.; Simon, G.A. (2020). Agronomic performance of grain sorghum cultivated in double rows space on Brazilian cerrado. Revista Caatinga. 33(2): 422-432.
Chi, Y.; Wilson, K.; Liu, Z.; Wu, X.; Shang, L.; Zhang, L.; Jing, H.; Hao, H. (2020). Vacuolar invertase genes SbVIN1 and SbVIN2 are differently associated with stem and kernel traits in sorghum (Sorghum bicolor). The Crop Journal. 8(1): 299-312.

Devore, J.L. (2006). Probabilidade e Estatística para Engenharia e Ciências. São Paulo: Thomson Pioneira. 706p.

Disasa, T.; Feyissa, T.; Admassu, B.; Fetene, M.; Mendu, V. (2018). Mapping of QTLs associated with brix and biomass-related traits in sorghum using SSR markers. Sugar Tech. 20(1): 275-285.

Empresa Brasileira de Pesquisa Agropecuária. (2012). BRS 506 o sorgo sacarino mais testado e plantado no Brasil. Sete Lagoas. Embrapa Milho e Sorgo. Disponível: $<$ https://www.embrapa.br/busca-depublicacoes/-/publicacao/937340/brs-506-osorgo-sacarino-mais-testado-e-plantado-nobrasil>. Acesso em: janeiro de 2021.

Ferreira, D.F. (2011). SISVAR: a computer statistical analysis system. Ciência e Agrotecnologia. 35(6): 1039-1042.

Godwin, J.J.; Venkatesan, H.; Sivamani, S. (2019). Renewable biofuel-a macro algal test study through its performance and emission analysis. International Conference on Electrical Energy Systems. 8(1): 1-6.

Hassan, M.U.; Chattha, M.U.; Barbanti, L.; Mahmood, A.; Chattha, M.B.; Khan, O.; Mirza, S.; Aziz, S.A.; Nawaz, M.; Aamer, M. (2020) Cultivar and seeding time role in sorghum to optimize biomass and methane yield under warm dry climate. Industrial Crops and Products. 145(1): 1-8. 
Isejima, E.M.; Costa, J.A.B.; Junior, D.I.S. (2002). Método de determinação de açúcares redutores aplicável no sistema de pagamento de cana-de-açúcar. Pesquisa Agropecuária Brasileira. 37(5): 729-734.

Leite, P.S.S.; Botelho, T.T.; Ribeiro, P.C.O.; Schaffert, R.E.; Parrella, R.A.C.; Nunes, J.A.R. (2019). Intrapopulation recurrent selection in sweet sorghum for improving sugar yield. Industrial Crops \& Products. 143(1): 1 - 8 .

Leite, M.J.H.; Gomes, A.D.V.; Santos, R.V. (2012). Cultivation of sorghum bicolor (L) Moench (S. vulgare pers) in seminarid. Revista verde de agroecologia e desenvolvimento sustentável. 7(1): 1-4.

Lima, M.T.V; Meireles, A.C.M.; Oliveira, C.W.; Nascimento, M.T.B. (2017). Koppen-Geiger and Thornthwaite climatic classification for the metropolitan region of the Cariri, Ceará. Revista Geama. 33(3): 136-143.

Masiero, G.; Lopes, H. (2008). Ethanol and biofuels as alternatives energetic sources: LatinAmerican e Asian perspectives. Revista Brasileira de Política Internacional. 51(2): 6079.

Mohammed, R.A.; Rasheed, I.M. (2020). Effect of irrigation water and organic fertilization on salinity level, soil salt accumulation and the growth of sorghum bicolor. Plant Archives. 20(1): 891-896.

Morey, S.R.; Hashida, Y.; Ohsugi, R.; Yamagishi, J.; Aoki, N. (2018). Evaluation of performance of sorghum varieties grown in Tokyo for sugar accumulation and its correlation with vacuolar invertase genes Sblnv 1 and Sblnv

2. Plant Production Science. 21(1): 328-338.
Nur, A.; Santos, S.B.; Syahruddin, K.; Efendy, R.; Lestari, E.G. (2019). Study of interaction between genetic source, harvest time and storage time in some mutant varieties of sweet sorghum to support future bioindustry development. IOP Conference Series: Earth and Environmental Science. 250(1): 1 - 10 .

Pimentel-Gomes, F. (2009). Curso de Estatística Experimental. (450p). 15 a edição. Piracicaba. FAELQ.

Prado, S.R.H.; Rezende, A.S.C.; Inácio, D.F.S.; Norberto, F.; Queiroz, J.N.S.M.; Melo, M.M.; Moreira, D.C.A.; Mendes, L.J.; Peixoto, J.L.; Cristeli, J.H. (2019). Feeding behavior of mangalarga marchador weanlings fed sorghum silage versus grass hay. Journal of Equine Veterinary Science. 75(1): 90-92.

R core team. (2016). R: A language and environment for statistical computing. $R$ Foundation for Statistical Computing. Disponível: <https://www.R-project.org/>. Acesso em: janeiro de 2021.

Santos, H.G.; Jacomine, P.K.T.; Anjos, L.H.C; Oliveira, V.A.; Lumbrearas, J.F.; Coelho, M.; Filho, J.C.A.; Almeida, J.A.; Oliveira, J.B.; Cunha, T.J.F. (2018). Sistema Brasileiro de Classificação de Solos. (353p). Ed. Brasília: EMBRAPA, 2018.

Silva, F.F.; Cavalcante, T.J.; Barbosa, K.P.; Castoldi, G.; Freiberger, M.B.; Silva, F.G. (2020). Potassic and nitrogen fertilization in a modern hybrid of sorghum for biomass production cultivated in an Oxisol. Australian Journal of Crop Science.14(1): 179-186.

Silva, T.M.; Oliveira, A.B.; Moura, J.G.; Trindade, B.F.L.; Oliveira, L.S.B. (2019). Potential of sweet sorghum juice as a source of ethanil for semi-arid regions: Cultivars and 
spacing arrangement effects. Sugar Tech. 21(1):

145-152.

Sobreira, F.M.; Fialho, G.S.; Sánchez, C.F.B.; Matta, F.P. (2009). Path analysis of postharvest in salad tomatoes type. Revista Facultad Nacional de Agronomia Medellín. 62(1): 49834988.
Stamenkovic, O.S., Siliveru, K., Velijkovic, V.B., Bankovic-Llic, I.B., Tasic, M.B., Ciampitti, I. A., Prasad, P.V.V. (2020). Production of biofuels from sorghum. Renewable and Sustainable Energy Reviews. 124(1): 1-24. 